\title{
Relación del consumo problemático de cannabis en la población joven de España con el riesgo percibido, los factores ambientales y los factores sociodemográficos
}

\author{
Relationship of problematic cannabis use among \\ youth in Spain with perceived risk, environmental \\ factors and sociodemographic factors
}

\author{
Jorge Arias-de la Torre*,**,***, Francisco José Eiroa-Orosa****, Antonio J. Molina**, \\ Esther Colell *****, Verónica Dávila-Batista******, Fernando Moreno-Montero****, \\ Noemí Robles $* * * * * *$, Jose M. Valderas $* * * * * * *$, Vicente-Martín $* *, * * *$. \\ * Institute of Psychiatry, Psychology and Neurosciences (IoPPN). King’s College London, Londres. Reino Unido. \\ ** Instituto de Biomedicina (IBIOMED). Universidad de León, León. España. \\ *** Centro de Investigación Biomédica en Red de Epidemiología y Salud Pública (CIBERESP), Madrid. España. \\ **** Departamento de Psicología Clínica y Psicobiología, Facultad de Psicología. Universidad de Barcelona, Barcelona. España. \\ ****** Consorcio Sanitario de Barcelona, Barcelona. España. \\ ******* eHealth Center. Universitat Oberta de Catalunya, Barcelona. España. \\ ******* Health Services and Policy Research Group. University of Exeter Medical School, Exeter. Reino Unido.
}

\section{Resumen}

La relación entre el consumo problemático de cannabis, el riesgo percibido y los factores socioambientales y sociodemográficos no es clara actualmente. Los objetivos del estudio son: describir los patrones de consumo de cannabis (problemático y no problemático) en la población joven de España y explorar como el consumo problemático se relaciona con el riesgo percibido, y los factores ambientales y sociodemográficos. Se llevó a cabo un diseño transversal basado en datos de la edición de 2015/2016 de la Encuesta Domiciliaria sobre Alcohol y Drogas (EDADES). La encuesta incluyó participantes de entre 15 y 35 años que habían consumido cannabis en al menos una ocasión durante el último año y que completaron el Cannabis Abuse Screening Test (CAST) $(\mathrm{N}=1674)$. Se consideró el consumo problemático (CAST >=7) como variable dependiente. Como variables independientes se consideraron el riesgo percibido, los factores ambientales (disponibilidad de la sustancia y exposición a situaciones de consumo) y los factores sociodemográficos. Se llevaron a cabo análisis descriptivos de los patrones de consumo y se realizaron modelos univariables y multivariables de Poisson. Todos los análisis se estratificaron por género. El consumo problemático fue más frecuente en hombres $(38,9 \%)$ que en mujeres $(23,2 \%)$. Mientras en hombres el consumo problemático se relacionó con factores ambientales y nivel educativo, en mujeres se asoció con riesgo percibido y desempleo.

\begin{abstract}
The relationship of problematic cannabis consumption with perceived risk, socioenvironmental and sociodemographic factors among youth in Spain is not well known. The aims of this study are: 1) to describe the patterns of cannabis consumption (problematic and non-problematic) in Spanish youth, and 2) to explore whether problematic cannabis consumption is related to perceived risk, environmental factors and individual sociodemographic characteristics. A cross-sectional design based on data from the 2015/16 Spanish Household Survey on Alcohol and Drugs (EDADES) was performed. Individuals between 15 and 35 years old having used cannabis during the last year with a complete Cannabis Abuse Screening Test (CAST) were included $(\mathrm{N}=1,674)$. Problematic consumption (CAST $>=7$ ) was considered as dependent variable. Perceived risk, environmental factors (availability of the substance and exposure to consumption situations) and sociodemographic factors were taken as independent variables. Descriptive analyses of consumption patterns were performed and univariable and multivariable Poisson regression models were done. All analyses were stratified by gender. Problematic cannabis consumption was more frequent among men $(38.9 \%)$ than among women $(23.2 \%)$. While among men, problematic use was related to environmental factors and educational level, among women it was associated with perceived risk and unemployment. Problematic cannabis consumption among Spanish youth is associated with
\end{abstract}


Dada la representatividad de los datos y la validez de las medidas, estos resultados podrían tener importantes implicaciones para el desarrollo de medidas preventivas contra el consumo problemático de cannabis

Palabras clave: Cannabis; Factores sociodemográficos; Factores ambientales; Riesgo percibido; Estudios de encuestas.

$\mathrm{H}$ oy en día, el cannabis es una de las sustancias recreativas más consumidas entre los jóvenes y una de las que mayor carga tiene a nivel mundial (Oficina de las Naciones Unidas contra la Droga y el Delito, 2016). Se estima que aproximadamente 17,2 millones de jóvenes europeos entre 15 y 34 años (aproximadamente 14,1\% de este segmento poblacional) ha consumido cannabis al menos una vez el último año, y alrededor de 9,8 millones de esos jóvenes entre 15 y 24 años, el 17,4 \% de este segmento poblacional (European Monitoring Centre for Drugs and Drug Addiction, 2017). En este contexto de alta prevalencia de consumo, es importante identificar qué factores pueden conducir al desarrollo de un patrón problemático de consumo entre los usuarios esporádicos. Se ha mostrado que los problemas asociados con el consumo de cannabis tienen una alta dependencia de los patrones de consumo (Silins et al., 2014), y el problemático se ha asociado de manera consistente con enfermedades severas y crónicas, además de tener consecuencias psicosociales negativas.

Varios estudios han intentado determinar qué factores están relacionados con el consumo problemático de cannabis (Colell, Sánchez-Niubò, Delclos, Benavides y Domingo-Salvany, 2015; Kokkevi, Richardson, Florescu, Kuzman y Stergar, 2007; Observatorio Español de las Drogas y las Toxicomanías, 2016; Redonnet, Chollet, Fombonne, Bowes y Melchior, 2012). Se han encontrado de manera consistente diferencias de género y edad, con un consumo problemático mayor en poblaciones de hombres más jóvenes (Arias-De la Torre et al., 2019; Rial et al., 2019). No obstante, esta literatura suele centrarse en grupos poblacionales específicos, como estudiantes o entornos de trabajo concretos, y en determinantes particulares como factores socioeconómicos y de personalidad.

Respecto de otros variables relacionados con el consumo problemático de cannabis, varios estudios han señalado una fuerte asociación con el riesgo percibido (Grevenstein, Nagy y Kroeninger-Jungaberle, 2015; Kirst, Mecredy, Borland y Chaiton, 2014; Salloum, Krauss, Agrawal, Bierut y Grucza, 2018). No obstante, muchos de estos estudios fueron realizados con muestras tomadas de la población general, que incluyen consumidores y no consumidores de sustancias. La realización de estudios independientes puede contribuir a mejorar nuestra comprensión de cómo el riesgo percibido podría resultar en el desarrollo de un different types of gender-related factors. Due to its representativeness at the population level and the validity of the measures, these results might have important implications on the development of prevention strategies targeted at problematic cannabis consumption.

Key words: Cannabis; Sociodemographic factors; Environmental factors; Perceived risk; Survey studies.

patrón problemático de consumo. Además, otros de los variables señalados como influyentes en el consumo problemático de cannabis son factores del entorno en el cual reside la persona, como disponibilidad de la sustancia y exposición social a situaciones de consumo, además de correlatos socioeconómicos (Kirst et al., 2014; Parnes, Smith y Conner, 2018). Saber cómo los factores ambientales se relacionan con el consumo problemático puede ser útil para proponer estrategias preventivas más efectivas y eficientes, adaptadas al contexto en el cual se implementarán. Sin embargo, hay un desconocimiento de la relación entre este tipo de variables y el consumo problemático. Respecto de los correlatos socioeconómicos, varios estudios han indicado que tanto la situación laboral como el nivel de estudios podrían estar relacionados con el consumo de cannabis entre los jóvenes (Colell et al., 2015; Teixidó-Compañó et al., 2018). De este modo, tener en cuenta estos variables al estudiar el consumo de cannabis podría contribuir a una mejor comprensión de los distintos patrones de consumo.

Por tanto, los objetivos de este estudio son: (1) describir los patrones de consumo de cannabis (problemático y no problemático) entre los jóvenes en España, y (2) someter a prueba si el consumo problemático de cannabis está relacionado con el riesgo percibido, factores ambientales y sociodemográficos.

\section{Material y métodos}

\section{Diseño y población del estudio}

Se llevó a cabo un diseño transversal basado en datos de la edición de 2015/2016 de la Encuesta Domiciliaria sobre Alcohol y Drogas (EDADES) (Observatorio Español de las Drogas y las Toxicomanías, 2016). EDADES es una encuesta representativa de la población española con edades entre 15 y 64 años. La muestra se eligió según un método de muestreo agrupado en tres etapas sin reemplazo, obteniendo una tasa de respuesta de 50,5\%. El cuestionario incluía información sobre el consumo de cannabis y los patrones del mismo, además de variables ambientales y sociodemográficas.

Como población del estudio se incluyeron los participantes menores de 36 años que habían consumido cannabis durante el último año en al menos una ocasión y que completaron el Cannabis Abuse Screening Test (CAST) ( $=$ 1674). Se excluyeron a las personas que carecían de datos 
sobre nivel de estudios $(n=2 ; 0,1 \%)$, situación laboral ( $n=51 ; 3,1 \%)$, riesgo percibido respecto del consumo regular $(n=10 ; 0,6 \%)$, percepción de la disponibilidad de las sustancias $(n=29 ; 1,7 \%)$ y exposición social a situaciones de consumo $(n=11 ; 0,7 \%)$. En total, se consideró una muestra de 1579 personas para el análisis.

\section{Variables de estudio}

Se consideró el consumo problemático según el CAST como variable dependiente. Este cuestionario es una herramienta de cribado válida y fiable para detectar el patrón problemático de consumo de cannabis (Cuenca-Royo et al., 2012). El cuestionario está compuesto de 6 ítems tipo Likert sobre la frecuencia de 5 eventos diferentes en los últimos 12 meses previos a la administración del mismo: consumo no recreativo de cannabis (2 ítems), problemas de memoria relacionadas con el consumo (1 ítem), preocupación de padres o amigos debido al consumo (1 ítem), intentos de abstinencia sin éxito ( 1 ítem) y otros posibles problemas relacionados con el consumo. Los ítems se puntúan en una escala tipo Likert de 0 («nunca») a 4 («con mucha frecuencia»). Se obtiene una puntuación total entre 0 y 24 al sumar cada ítem de la escala. El punto de corte para consumo problemático de cannabis se fijó en 7 o más ( $\mathrm{AUC}=0,83)$ como propuso un estudio de validación poblacional en España (Cuenca-Royo et al., 2012).

Como variables explicativas, se consideraron el riesgo percibido, factores ambientales (disponibilidad de la sustancia y exposición a situaciones de consumo) y factores sociodemográficos. El riesgo percibido se valoró con un ítem tipo Likert con una escala de respuestas de cuatro puntos, desde 1 («ningún problema») a 4 («muchos problemas») respecto del consumo de cannabis al menos una vez por semana. La disponibilidad de las sustancias se valoró con un ítem tipo Likert con una escala de respuestas de cuatro puntos, desde 1 («casi imposible») a 4 («muy fácilmente») respecto de la dificultad para obtener la sustancia en un período de 24 horas. Debido a que había pocas personas en los niveles de las variables «casi imposible» $\mathrm{y}$ «difícil», estas categorías se colapsaron en «difícil». La exposición a situaciones de consumo se valoró con un ítem tipo Likert con una escala de respuestas de cuatro puntos, desde 1 («habitualmente») a 4 («nunca») respecto de la frecuencia en la cual se encontraba a personas de su entorno cercano fumando cannabis. Además, se consideraron los siguientes factores sociodemográficos: género, edad como variable dicotómica (de 16 a 25 años y de 26 a 35 años), nivel de estudios (universitarios, secundarios/bachillerato y primarios/sin estudios) y situación laboral (empleado, desempleado, estudiante).

\section{Análisis estadístico}

Realizamos un análisis descriptivo de la distribución del consumo problemático de cannabis entre los factores expli- cativos por género. Evaluamos las diferencias mediante las pruebas de del chi cuadrado y exacta de Fisher. Después, realizamos modelos de regresión bivariables y multivariables de Poisson para probar la asociación entre los factores explicativos y la variable dependiente. Obtuvimos de estos modelos el Riesgo Relativo Crudo (RR), el Riesgo Relativo ajustado (aRR) y sus Intervalos de Confianza de $95 \%$ (95\% IC). Todos los modelos multivariable se ajustaron para todas las variables explicativas. La bondad de ajuste de los modelos multivariable se valoró mediante el test de chi cuadrado. Además, se verificó la ausencia de multicolinearidad y de interacciones entre las variables. Los valores $p$ para la significancia estadística global para cada variable se obtuvieron mediante la prueba de Wald. Todos los modelos se estratificaron por género en base al marco teórico propuesto por estudios anteriores (Arias-De la Torre et al., 2019; Colell et al., 2015; Kirst et al., 2014; Redonnet et al., 2012), y tuvieron en cuenta los pesos derivados del diseño muestral complejo. Usamos los programas informáticos Stata v.14 para todos los análisis estadísticos (StataCorp, 2015).

\section{Resultados}

La Tabla 1 muestra las características de la muestra del estudio y el patrón de consumo de cannabis por género. Puede observarse que el consumo problemático de cannabis es más frecuente en hombres $(38,9 \%)$ que en mujeres $(23,2 \%)$. Entre los hombres, hallamos diferencias estadísticamente significativas en los patrones de consumo en todas las variables explicativas, exceptuando edad. No obstante, entre las mujeres hallamos estas diferencias en riesgo percibido, nivel de estudios y situación laboral.

Además, la Tabla 2 muestra la relación entre el consumo problemático de cannabis y todas las variables explicativas. Tras el ajuste, la disponibilidad de la sustancia, la exposición a situaciones de consumo y el nivel de estudios estuvieron significativamente relacionados con el consumo problemático $(p<, 05)$ entre los hombres. En cambio, entre las mujeres solamente el riesgo percibido estuvo relacionado con este patrón de consumo. Además, teniendo en cuenta las categorías específicas para cada una de las variables incluidas, se mostró un mayor riesgo de consumo problemático de cannabis para hombres que solían tener una exposición a situaciones de consumo (aRR: 1,42; 95 \% IC: 1,02-1,98 tomando «nunca expuesto» como categoría de referencia) y entre los de un nivel de estudios primarios o analfabetos (aRR: 1,68; $95 \%$ IC: 1,22-2,30 tomando estudios universitarios como categoría de referencia. Entre las mujeres, se mostró un mayor riesgo de consumo problemático de cannabis entre aquellas que no percibieron «ningún problema» relacionado con su consumo (aRR: 1,96; 95 \% IC: 1,01-3,80 tomando muchos problemas como categoría de referencia) y entre las desempleadas (aOR: 1,95; 95 \% IC: 1,27-2,98 tomando empleada como categoría de referencia. 
Relación del consumo problemático de cannabis en la población joven de España con el riesgo percibido, los factores ambientales y los factores sociodemográficos

Tabla 1. Características de la muestra del estudio y diferencias en los patrones de consumo de cannabis entre los jóvenes en España por género. Encuesta sobre Alcohol y Drogas en España (EDADES) 2015-2016.

\begin{tabular}{|c|c|c|c|c|c|c|c|c|c|c|}
\hline & \multicolumn{5}{|c|}{ Hombres $(\mathrm{N}=1117)$} & \multicolumn{5}{|c|}{ Mujeres $(\mathrm{N}=462)$} \\
\hline & \multicolumn{2}{|c|}{$\begin{array}{c}\text { Usuarios no } \\
\text { problemáticos } \\
(\mathrm{n}=683 ; 61,1 \%)\end{array}$} & \multicolumn{2}{|c|}{$\begin{array}{c}\text { Usuarios } \\
\text { problemáticos } \\
(n=434 ; 38,9 \%)\end{array}$} & \multirow[b]{2}{*}{ valor $p$} & \multicolumn{2}{|c|}{$\begin{array}{c}\text { Usuarios no } \\
\text { problemáticos } \\
(\mathrm{n}=355 ; 76,8 \%)\end{array}$} & \multicolumn{2}{|c|}{$\begin{array}{c}\text { Usuarios } \\
\text { problemáticos } \\
(\mathrm{n}=107 ; 23,2 \%)\end{array}$} & \multirow[b]{2}{*}{ valor $p$} \\
\hline & $\mathbf{n}$ & $\%$ & $\mathbf{n}$ & $\%$ & & $\mathbf{n}$ & $\%$ & $\mathbf{n}$ & $\%$ & \\
\hline Riesgo percibido & & & & & $<, 001$ & & & & &, 008 \\
\hline Muchos problemas & 107 & 15,7 & 57 & 13,1 & & 56 & 17,8 & 10 & 9,4 & \\
\hline Algunos problemas & 179 & 26,2 & 74 & 17,1 & & 101 & 28,5 & 25 & 23,4 & \\
\hline Pocos problemas & 253 & 37,0 & 163 & 37,6 & & 127 & 35,8 & 34 & 31,8 & \\
\hline Ningún problema & 144 & 21,1 & 140 & 32,3 & & 71 & 20,0 & 38 & 35,5 & \\
\hline Disponibilidad (en 24 horas) & & & & & $<, 001$ & & & & &, 271 \\
\hline Difícil & 49 & 7,2 & 21 & 4,8 & & 23 & 6,5 & 5 & 4,7 & \\
\hline Relativamente fácil & 182 & 26,7 & 69 & 15,9 & & 103 & 29,0 & 24 & 22,4 & \\
\hline Muy fácil & 452 & 66,2 & 344 & 79,3 & & 229 & 64,5 & 78 & 72,9 & \\
\hline Exposición a situaciones & & & & & $<, 001$ & & & & & .438 \\
\hline Nunca & 92 & 13,5 & 34 & 7,8 & & 37 & 10,4 & 9 & 8,4 & \\
\hline Rara vez & 125 & 18,3 & 57 & 13,1 & & 60 & 16,9 & 12 & 11,2 & \\
\hline Con frecuencia & 249 & 36,5 & 157 & 36,2 & & 130 & 36,6 & 44 & 41,1 & \\
\hline Habitualmente & 217 & 31,8 & 186 & 42,9 & & 128 & 36,1 & 42 & 39,3 & \\
\hline Nivel de estudios & & & & & $<, 001$ & & & & &, 022 \\
\hline Universitarios & 95 & 13,9 & 45 & 10,4 & & 83 & 23,4 & 14 & 13,1 & \\
\hline Secundarios o bachillerato & 525 & 76,9 & 301 & 69,6 & & 251 & 70,7 & 81 & 75,7 & \\
\hline Primarios o analfabeto & 63 & 9,2 & 87 & 20,1 & & 21 & 5,9 & 12 & 11,2 & \\
\hline Situación laboral & & & & & $<, 001$ & & & & &, 010 \\
\hline Empleado & 309 & 45,2 & 194 & 44,7 & & 173 & 48,7 & 45 & 42,1 & \\
\hline Desempleado/a & 166 & 24,3 & 150 & 34,6 & & 59 & 16,6 & 32 & 29,9 & \\
\hline Estudiante & 208 & 30,5 & 90 & 20,7 & & 123 & 34,7 & 30 & 28,0 & \\
\hline Edad & & & & &, 086 & & & & &, 670 \\
\hline Entre $16-25$ años & 304 & 44,5 & 216 & 49,8 & & 151 & 42,5 & 48 & 44,9 & \\
\hline Entre $26-35$ años & 379 & 55,5 & 218 & 50,2 & & 204 & 57,5 & 59 & 55,1 & \\
\hline
\end{tabular}

Nota. N: muestra total analizada; \%: porcentaje por columna; $95 \%$ IC: $95 \%$ Intervalo de Confianza.

Valor $p$ : Pruebas de del chi cuadrado y exacta de Fisher. Usuarios no problemáticos: CAST < 7; usuarios problemáticos CAST $>7$.

\section{Discusión}

Los resultados muestran que, hoy en día, el consumo problemático de cannabis entre los jóvenes en España tiene una prevalencia alta, especialmente entre los hombres. Es más, mientras que en los hombres el consumo problemático parece estar relacionado con factores ambientas y nivel de estudios, entre las mujeres el consumo problemático de cannabis parece estar relacionado con el riesgo percibido y el desempleo. Con un enfoque en los factores relacionados con el consumo problemático, debería señalarse que la prevalencia de consumo problemático es más alta en hombres que en mujeres, y que, para los hombres, los factores externos y contextuales parecen estar estrechamente relacionados con este patrón de consumo. Entre las mujeres, no obstante, los factores internos y personales parecen cobrar una relevancia especial. Estos resultados son coherentes con los obtenidos en algunos estudios anteriores (Bonar et al., 2017; Foster, Jeffries, Zvolensky y Buckner,
2016; Haug, Núñez, Becker, Gmel y Schaub, 2014; Kirst et al., 2014), y pueden explicarse en parte por diferencias de género en las estrategias de afrontamiento al estrés entre los jóvenes. Mientras que en países con valores tradicionales familiares, como es el caso de España, se espera de los hombres jóvenes que tengan un estilo de afrontamiento orientado hacia lo externo y puedan estar más motivados por el impulso y la oportunidad, se espera de las mujeres jóvenes que tengan un estilo de afrontamiento orientado hacia lo interno y que más bien adapten sus actitudes según experiencias conductuales (Casajuana Kögel et al., 2021; Foster et al., 2016; García-Sánchez et al., 2016). Por tanto, como estudios anteriores han sugerido (Casajuana Kögel et al., 2021; Haug et al., 2014; Kokkevi et al., 2007; López-Pelayo, Miquel De Montagut, Casajuana Kögel y Balcells Oliveró, 2018), ofrecer estrategias adaptativas para afrontar el estrés (centradas en factores externos en hombres y en factores internos en mujeres) podría ser un en- 
Tabla 2. Relación entre el consumo problemático de cannabis con riesgo percibido, la disponibilidad de la sustancia, la exposición a situaciones de consumo y los factores sociodemográficos en los jóvenes en España. Análisis bivariable y multivariable. Encuesta sobre Alcohol y Drogas en España (EDADES) 2015-2016.

\begin{tabular}{|c|c|c|c|c|c|c|c|c|}
\hline & & Hom & res & & & & & \\
\hline & RR (95 \% IC) & $\mathrm{p}$ & aRR (95\% IC) & $\mathrm{p}$ & RR (95 \% IC) & p & aRR (95 \% IC) & $\mathrm{p}$ \\
\hline Riesgo percibido & & ,006 & &, 067 & & ,007 & & 022 \\
\hline Muchos problemas & 1,00 & & 1,00 & & 1,00 & & 1,00 & \\
\hline Algunos problemas & $0,66(0,48-0,91)$ & & $0,67(0,49-0,91)$ & & $1,17(0,56-2,45)$ & & $1,21(0,59-2,46)$ & \\
\hline Pocos problemas & $0,94(0,73-1,22)$ & & $0,91(0,71-1,18)$ & & $1,28(0,63-2,60)$ & & $1,31(0,65-2,64)$ & \\
\hline Ningún problema & $1,21(0,94-1,56)$ & & $1,06(0,82-1,36)$ & & $2,27(1,15-4,50)$ & & $1,96(1,01-3,80)$ & \\
\hline Disponibilidad (en 24 horas) & &, 001 & &, 008 & & & & \\
\hline Difícil & 1,00 & & 1,00 & & 1,00 & 629 & 1,00 & ,897 \\
\hline Relativamente fácil & $1,03(0,62-1,02)$ & & $1,04(0,63-1,69)$ & & $0,63(0,26-1,65)$ & & $0,65(0,30-1,41)$ & \\
\hline Muy fácil & $1,57(0,98-2,50)$ & & $1,41(0,90-2,21)$ & & $0,92(0,39-2,18)$ & & $0,81(0,41-1,60)$ & \\
\hline Exposición a situaciones & & 001 & &, 023 & & 091 & & ,245 \\
\hline Nunca & 1,00 & & 1,00 & & 1,00 & & 1,00 & \\
\hline Rara vez & $1,05(0,70-1,58)$ & & $1,11(0,75-1,64)$ & & $0,66(0,26-1,65)$ & & $0,67(0,27-1,64)$ & \\
\hline Con frecuencia & $1,24(0,87-1,76)$ & & $1,21(0,86-1,71)$ & & $1,39(0,65-2,97)$ & & $1,24(0,59-2,63)$ & \\
\hline Habitualmente & $1,56(1,11-2,20)$ & & $1,42(1,02-1,98)$ & & $1,36(0,63-2,94)$ & & $1,07(0,51-2,22)$ & \\
\hline Nivel de estudios & & $<, 001$ & & $<, 001$ & & ,114 & & 203 \\
\hline Universitarios & 1,00 & & 1,00 & & 1,00 & & 1,00 & \\
\hline Secundarios o bachillerato & $1,17(0,87-1,56)$ & & $1,14(0,86-1,52)$ & & $1,47(0,80-2,68)$ & & $1,38(0,75-2,52)$ & \\
\hline Primarios o analfabeto & $1,91(1,39-2,61)$ & & $1,68(1,22-2,30)$ & & $1,79(0,81-3,95)$ & & $1,48(0,65-3,38)$ & \\
\hline Situación laboral & &, 049 & & , 165 & & .557 & & .536 \\
\hline Empleado/a & 1,00 & & 1,00 & & 1,00 & & 1,00 & \\
\hline Desempleado/a & $1,19(0,99-1,43)$ & & $1,09(0,91-1,31)$ & & $2,21(1,45-3,36)$ & & $1,95(1,27-2,98)$ & \\
\hline Estudiante & $0,75(0,59-0,95)$ & & $0,79(0,61-1,01)$ & & $1,10(0,68-1,76)$ & & $1,09(0,65-1,83)$ & \\
\hline Edad & & 128 & &, 222 & & ,764 & &, 545 \\
\hline Entre 16-25 años & 1,00 & & 1,00 & & 1,00 & & 1,00 & \\
\hline Entre $26-35$ años & $0,88(0,74-1,04)$ & & $0,97(0,81-1,15)$ & & $0,94(0,64-1,38)$ & & $0,91(0,61-1,35)$ & \\
\hline
\end{tabular}

Nota. RR: Riesgo Relativo Crudo, aRR: Riesgo Relativo ajustado (aRR) 95 \% IC: 95 \% Intervalo de Confianza. p: valor p de la prueba de Wald.

Los modelos multivariable se ajustaron para todas las variables explicativas y los factores demográficos. Todos los análisis se realizaron teniendo en cuenta los pesos derivados del diseño muestral complejo. Todos los modelos multivariable tuvieron un valor $p$ >,05 en la prueba de bondad de ajuste chi cuadrado.

foque efectivo para afrontar el consumo problemático de cannabis. Sin embargo, para explorar la posible influencia bidireccional entre las variables explicativas y el consumo problemático de cannabis, podría ser especialmente relevante establecer medidas de prevención específicas centradas en la causa del consumo problemático, como es el caso de desempleo en las mujeres aunque, como han sugerido estudios longitudinales anteriores, la dirección de esta relación no está clara (Popovici y French, 2014).

Respecto de la direccionalidad de las relaciones entre las variables, debemos señalar que, como han sugerido estudios anteriores, es posible que la relación hallada entre el riesgo percibido y el consumo problemático sea bidireccional (Salloum et al., 2018). Los resultados de este estudio confirman el principio que «la conducta precede la actitud». En este sentido, podría haber asociaciones longitudinales recíprocas entre el consumo de cannabis y el riesgo percibido, con una asociación más fuerte entre el consumo de cannabis y un menor riesgo percibido subsecuente. Podrían ser valiosos más estudios con población española con datos longitudinales para obtener una mejor comprensión de esta relación.

Respecto de los métodos usados, debemos señalar que nuestro estudio es uno de los más grandes realizados en Europa con el fin de valorar el consumo problemático de cannabis. Además, usamos una muestra representativa a nivel de país y una herramienta válida y fiable diseñada específicamente para este fin. Los estudios anteriores no usaron muestras representativas ni herramientas válidas y fiables para determinar los patrones de consumo. La combinación de este tipo de muestras con el uso de herramientas con propiedades métricas adecuadas, como el cuestionario CAST (Cuenca-Royo et al., 2012), podrían aportar estimaciones más precisas del consumo problemático, y además ayudar a mejorar la fiabilidad al determinar los factores específicos relacionados con los diferentes patrones. 
Hemos de abordar varias de las limitaciones de este estudio. Primero, su diseño transversal impide determinar la dirección causal de la relación entre el consumo problemático y los factores explicativos. Sin embargo, este diseño podría ser el adecuado para identificar posibles factores relacionados con el patrón de consumo. Segundo, debemos señalar que la sensibilidad y la especificidad no son óptimas. A pesar de ello, el cuestionario CAST ha mostrado propiedades métricas adecuadas (Cuenca-Royo et al., 2012). Es más, es la primera vez que esta herramienta se ha incluido en EDADES. Por tanto, nuestros resultados pueden servir de referencia para una valoración válida y fiable del consumo problemático de cannabis y sus factores relacionados. Por último, hay algunas limitaciones inherentes a la naturaleza autoinformada de los datos (i.e., algunas personas pueden ser reticentes a responder a ciertas preguntas sobre su consumo de sustancias). Sin embargo, dado que el cuestionario fue anónimo, podemos considerar a priori que las respuestas fueron imparciales. De todas maneras, la tasa de respuesta fue de $50,5 \%$, y la representatividad a nivel poblacional estuvo garantizada (Observatorio Español de las Drogas y las Toxicomanías, 2016). Por tanto, consideramos que la población estudiada es adecuada para el desarrollo de los objetivos propuestos.

En conclusión, nuestro estudio muestra que el consumo problemático de cannabis entre los jóvenes en España es alto, especialmente entre los hombres, y que podría estar relacionad con distintos tipos de factores dependientes del género. Es más, estos resultados pueden servir como punto de partida para más estudios dentro y fuera de Europa y, también, pueden ayudar a identificar los factores asociados al desarrollo de patrones de consumo problemático de cannabis.

\section{Reconocimientos}

Agradecemos al CIBERESP por la financiación aportada para este estudio.

\section{Papel de la fuente de financiación}

Este estudio fue financiado por el Centro de Investigación Biomédica en Red de Epidemiología y Salud Pública (CIBERESP) como parte de las ayudas para estancias de corta duración concedida a Jorge Arias-De la Torre en 2017 y 2018.

\section{Conflicto de intereses}

Los autores declaran la inexistencia de conflictos de intereses en relación al estudio, su autoría, y/o la publicación de este manuscrito.

\section{Referencias}

Arias-De la Torre, J., Fernández-Villa, T., Molina, A. J., Amezcua-Prieto, C., Mateos, R., Cancela, J. M., ... Martín, V. (2019). Drug use, family support and related factors in university students. A cross-sectional study based on the uniHcos Project data. Gaceta Sanitaria, 33, 141-147. doi:10.1016/j.gaceta.2017.10.019.

Bonar, E. E., Goldstick, J. E., Collins, R. L., Cranford, J. A., Cunningham, R. M., Chermack, S. T., ... Walton, M. A. (2017). Daily associations between cannabis motives and consumption in emerging adults. Drug and Alcohol Dependence, 178, 136-142. doi:10.1016/j.drugalcdep.2017.05.006.

Casajuana Kögel, C., López-Pelayo, H., Oliveras, C., Colom, J., Gual, A. y Balcells-Oliveró, M. M. (2021). The relationship between motivations for cannabis consumption and problematic use. Adicciones, 33, 31-41. doi:10.20882/adicciones.1221.

Colell, E., Sánchez-Niubò, A., Delclos, G. L., Benavides, F. G. y Domingo-Salvany, A. (2015). Economic crisis and changes in drug use in the Spanish economically active population. Addiction, 110, 1129-1137. doi:10.1111/ add.12923.

Cuenca-Royo, A. M., Sánchez-Niubó, A., Forero, C. G., Torrens, M., Suelves, J. M. y Domingo-Salvany, A. (2012). Psychometric properties of the CAST and SDS scales in young adult cannabis users. Addictive Behaviors, 37, 709715. doi:10.1016/j.addbeh.2012.02.012.

European Monitoring Centre for Drugs and Drug Addiction. (2017). European Drug Report 2017: Trends and Developments. Luxembourg: Publications Office of the European Union.

Foster, D. W., Jeffries, E. R., Zvolensky, M. J. y Buckner, J. D. (2016). The interactive influence of cannabis-related negative expectancies and coping motives on cannabis use behavior and problems. Substance Use E Misuse, 51, 1504-1511. doi:10.1080/10826084.2016.1188947.

García-Sánchez, S., Matalí, J. L., Martín-Fernández, M., Pardo, M., Lleras, M., Castellano-Tejedor, C. y Haro, J. M. (2016). Internalizing and externalizing personality and subjective effects in a sample of adolescent cannabis users. Adicciones, 28, 231-241. doi:10.20882/adicciones.887.

Grevenstein, D., Nagy, E. y Kroeninger-Jungaberle, H. (2015). Development of risk perception and substance use of tobacco, alcohol and cannabis among adolescents and emerging adults: evidence of directional influences. Substance Use E्F Misuse, 50, 376-386. doi:10.3109/10826 084.2014.984847.

Haug, S., Núñez, C. L., Becker, J., Gmel, G. y Schaub, M. P. (2014). Predictors of onset of cannabis and other drug use in male young adults: results from a longitudinal study. BMC Public Health, 14, 1202. doi:10.1186/14712458-14-1202. 
Kirst, M., Mecredy, G., Borland, T. y Chaiton, M. (2014). Predictors of substance use among young adults transitioning away from high achool: A narrative review. Substance Use Eं Misuse, 49, 1795-1807. doi:10.3109/108 26084.2014.933240.

Kokkevi, A., Richardson, C., Florescu, S., Kuzman, M. y Stergar, E. (2007). Psychosocial correlates of substance use in adolescence: A cross-national study in six European countries. Drug and Alcohol Dependence, 86, 67-74. doi:10.1016/j.drugalcdep.2006.05.018.

López-Pelayo, H., Miquel De Montagut, L., Casajuana Kögel, C. y Balcells Oliveró, M. (2018). Post-truth Cannabis use: back to evidence-based medicine. Adicciones, 30, 237-242. doi:10.20882/adicciones.1095.

Observatorio Español de las Drogas y las Toxicomanías. (2016). Encuesta sobre alcohol y otras drogas en España 2015/2016. Madrid: Ministerio de Sanidad, Servicios Sociales e Igualdad.

Oficina de las Naciones Unidas contra la Droga y el Delito. (2016). World Drug Report 2016. New York: United Nations publication, Sales No. E.16.XI.7.

Parnes, J. E., Smith, J. K. y Conner, B. T. (2018). Reefer madness or much ado about nothing? Cannabis legalization outcomes among young adults in the United States. International Journal of Drug Policy, 56, 116-120. doi:10.1016/j.drugpo.2018.03.011.

Popovici, I. y French, M. T. (2014). Cannabis Use, Employment, and Income: Fixed-Effects Analysis of Panel Data.
The Journal of Behavioral Health Services E Research, 41, 185-202. doi:10.1007/s11414-013-9349-8.

Redonnet, B., Chollet, A., Fombonne, E., Bowes, L. y Melchior, M. (2012). Tobacco, alcohol, cannabis and other illegal drug use among young adults: The socioeconomic context. Drug and Alcohol Dependence, 121, 231239. doi:10.1016/j.drugalcdep.2011.09.002.

Rial, A., Burkhart, G., Isorna, M., Barreiro, C., Varela, J. y Golpe, S. (2019). Cannabis use among adolescents: Risk pattern, implications and possible explanatory variables. Adicciones, 31, 64-77. doi:10.20882/adicciones.1212.

Salloum, N. C., Krauss, M. J., Agrawal, A., Bierut, L. J. y Grucza, R. A. (2018). A reciprocal effects analysis of cannabis use and perceptions of risk. Addiction, 113, 10771085. doi:10.1111/add.14174.

Silins, E., Horwood, L. J., Patton, G. C., Fergusson, D. M., Olsson, C. A., Hutchinson, D. M.,... Mattick, R. P. (2014). Young adult sequelae of adolescent cannabis use: an integrative analysis. Lancet Psychiatry, 1, 286-293. doi:10.1016/S2215-0366(14)70307-4.

StataCorp. (2015). Stata Statistical Software: Release 14. College Station, TX: StataCorp LLC.

Teixidó-Compañó, E., Espelt, A., Sordo, L., Bravo, M. J., Sarasa-Renedo, A., Indave, B. I.,... Brugal, M. T. (2018). Differences between men and women in substance use: the role of educational level and employment status. Gaceta Sanitaria, 32, 41-47. doi:10.1016/j.gaceta.2016.12.017. 
